\title{
Post-modern-east ou comment peut-on être «post-moderniste sans post-modernité » et sans Lyotard ? ${ }^{1}$
}

\section{ALEXANDRU MATEI}

\begin{abstract}
The Post-Modern East, or How Can We Be 'Post-Modern without Postmodernity' and without Lyotard? Despite the idea of the universality of 'postmodernism' as a new stage in the Western World, it is now clear that the term was coined, launched, adopted or rejected differently in different places, along local historical lines. Hence, we have not only an American and a European postmodernism, but also an East European postmodernism, what we shall call the Post-Modern East. We delineate its characteristics based on a survey that looked at how East European cultures adopted and discussed postmodernism around the moment that their socialist regimes were collapsing. We focus the analysis on a particular but synthetising version of the 'postmodern', specifically that of Lyotard. We hold that Lyotard is one of the few intellectuals who succeeded in thinking of politics, sociology, epistemology and aesthetics as tying together to form 'postmodernity'; and that a few European intellectuals were ready to think of 'postmodernity' an epistemic challenge, beyond the distinction between soft and hard sciences. A fortiori, Eastern European cultures seized 'postmodernism' as an American fetish and identified the breakdown of totalitarianism as the achievement of happy 'postmodernisation'. Thirty years later, these countries have realised that by embracing a certain version of 'postmodern', as they had done by the end of the 1980s, was generally a mimetic utopian gesture that needs revaluation.
\end{abstract}

Keywords: Postmodernism/Postmodernity; Jean-François Lyotard; content/ form; Eastern Europe; Mircea Martin

Les temps qui courent peuvent être saisis en versions colorisées dès les années 1960 dans à peu près toute l'Europe, et il est vrai que la télévision se rapproche encore plus des masses quand elle diffuse des images en couleurs. Mais la « terreur » qu'évoque Lyotard à propos des jeux de langage imposés par les

1 Ce texte est publié dans le cadre du projet 21-AUF/01.03.2019, financé par l'AUF par l'IFA, « Transferts culturels et champs intellectuels internationaux sous le regime communiste. Modernite et antimodernite a l'Est et a l'Ouest: France, Roumanie, Serbie. » 
institutions discursives modernes (Lyotard 1979a : 90), dans laquelle nous reconnaissons la référence à Paulhan (« Les Fleurs de Tarbes ou la terreur dans les lettres $\gg$, titre qui date de 1941), ne reviendra plus : les jeux de langage sont entrés dans l'ère du « brouhaha » (Ruffel : 2016).

Le terme « postmoderne » est né aux Etats-Unis, on le sait. Une fois passé en Europe, le « postmodernisme » (ou bien la « postmodernité », ou encore le « postmoderne ») s'associe à une réflexivité discursive accrue, dont l'interprétation de l'Histoire moderne comme récit (ou bien comme métarécit) n'est que l'un des effets qui portent le plus à conséquence dans le champ de la théorie. Mais si cette réflexivité prend naissance dans une société qui, depuis la fin des années 1960, assiste à un certain nombre de changements sociaux, économiques et d'éthos (et bientôt politiques), rien ne prépare, apparemment, son passage au-delà du Rideau de fer, dans une Europe soumise encore à des régimes socio-économiques plus ou moins inspirés de Marx et de Lénine.

Notre étude se propose de retracer l'histoire de la réception du terme en Europe de l'Est et notamment en Roumanie, selon sa version synthétique, qui noue politique, épistémologie et esthétique, de Jean-François Lyotard. On retrouvera ainsi, en creux, les contours d'une fausse réflexivité (ou d'une réflexivité de la «mauvaise conscience ») dont les limites, invisibles alors, se laissent expliquer aujourd'hui précisément à la lumière du partage en vigueur tout au long de l'époque soviétique, entre un domaine « esthétique » des formes, et un domaine « idéologique » de l'Histoire et de la Vérité.

Par-delà la « postmodernité » nord-américaine, sociale, économique et artistique dont on discute dès les années 1960 surtout sur le mode du constat (voir Howe 1959), la « condition postmoderne » de Lyotard proposait une évaluation critique des « savoirs » élaborée à l'aide d'analyses pragmatiques, qui menaçait à la fois le sérieux du discours politique officiel des régimes socialistes et celui du pacte mutuel entre les intellectuels humanistes et les représentants du pouvoir politique, un pacte assurant l'autonomie des discours sur l'art et l'esthétique à condition que leurs auteurs ne se lancent pas dans la critique sociale et politique.

Si de jeunes intellectuels roumains anglophones se sont faits, dès les années 1980, les passeurs du débat postmoderne américain, ils ont peu pensé à ce que Lyotard appelle un «transfert » de discours, autrement dit aux conditions pragmatiques qui sont celles du « postmoderne » transplanté en Roumanie et dans l'Europe de l'Est. L'expérience historique de l'Est nous montre, en rétrospection, comment la charnière de 1989 a contribué à maintenir séparés deux « postmodernismes »: d'une part, un postmodernisme esthétique (et une postmodernité d'éthos) qui a mobilisé les esprits artistiques dès avant la chute des régimes totalitaires, et d'autre part un postmodernisme « social », 
MATEI

ou bien le postmodernisme « réel », auprès duquel les mêmes intellectuels étaient démunis. Cet apparent paradoxe se laisse voir à la lecture d'un des plus connus essais écrits en Roumanie à propos du moment postmoderne : « D'un postmodernisme sans rivages et d'un postmodernisme sans postmodernité » (Martin 1995 : 3-13), que nous allons présenter et discuter rapidement à la fin de ce texte.

\section{La Condition postmoderne, par-delà les sciences humaines et les sciences dures}

Le rapport que Jean-François Lyotard rédige pour le Conseil des Universités du Gouvernement de Québec porte le titre Le Problème du savoir dans les sociétés industrielles les plus développées. Dès l'avertissement, le philosophe précise que, comme il n'est pas un expert, « qui sait ce qu'il sait et ce qu'il ne sait pas parce qu'il sait ce qu'est savoir dans sa partie $\gg$ (Lyotard 1979a : 2), il pourra ne pourra formuler que des «interrogations » à propos de tout ce que les experts sont appelés à offrir des réponses. C'est depuis ce socle socratique, voire montaignien, du « que sais-je ? » (Lyotard voit Montaigne «postmoderne » dans son article visant à donner pourtant une réponse à la question Qu'est-ce que le postmoderne? - Lyotard 1986 : 86) qu'il entend écrire. C'est une attitude qui l'installe déjà dans une position de liberté et en quelque sorte d'« irresponsabilité » par rapport à un certain type d'attentes immédiatement lucratives, auxquelles satisfont les experts. Ainsi enchaîne-t-il :

Le savoir est et sera produit pour être vendu, et il est et sera consommé pour être valorisé dans une nouvelle production : dans les deux cas, pour être échangé. (...) Dans l'âge post-industriel et post-moderne la science conservera et sans doute renforcera encore son importance dans la batterie des capacités productives des Etats-nations. Cette situation est même l'une des raisons qui font penser que l'écart avec les pays en voie de développement ne cessera pas à l'avenir de s'élargir (Lyotard 1979a : 6)

Dans l'avant-propos, Lyotard emploie déjà le terme « post-moderne » (《dans l'âge dit post-moderne $\gg$ ) et offre, en note de fin d'ouvrage, une liste bibliographique justificative : A. Touraine, La société post-industrielle (1969) ; D. Bell, The Coming of Post-Industrial Society (1973); Ihab Hassan, The Dismemberment of Orpheus (1971) ; M. Benamou \& Ch. Caramello (editors), Performance in Postmodern Culture (1977) ; M. Kohler, Postmodernismus : ein begriff geschichtlicher Ueberblick, dans la revue Amerikastudien 22, 1 (1977) (Lyotard 1979a : 94). 
Post-modern-east ou comment peut-on être « post-moderniste sans post-modernité »

Il assimile donc post-industriel et post-moderne, mais « La Société postindustrielle $\gg$ est le seul apport terminologique francophone dans cette liste bibliographique. Il parle d'informatisation de la société, de savoir expertisé, de calcule et d'efficience. Il parle ensuite des rapports entre gouvernement (on dirait « gouvernance » aujourd'hui), savoir et société, sur le mode pragmatique. Il constate la disparition de la «lutte des classes » et la fin de deux idées de sociétés : une société-machine qu'il s'agit de faire fonctionner par la science « objective » (« positiviste »), et une société scindée entre le statu quo et sa critique (dont rend compte le discours « critique »). Or, cette pensée oppositionnelle ne correspond plus, dit-il, aux « modes les plus vivaces du savoir postmoderne $\gg$ (Lyotard 1979b : 20). On pourrait dire que c'est dans le constat de cette inadéquation qu'est posé le principe de la « postcritique » contemporaine qui entend en finir avec le scepticisme soupçonneux typique de ce que Paul Ricœur appelait «l'herméneutique de la suspicion », selon Rita Felski (Felski 2015).

Que la « condition postmoderne » concerne le savoir, mais aussi le langage qui le manifeste - et, à travers le langage, tous les pans du monde (société, culture, économie, en ce qu'ils sont le prétexte d'une nomination) - c'est ce que constate, une décennie plus tard, un philosophe hollandais, Ernst van Alphen. Il soutient que

une perspective monodisciplinaire sur le postmodernisme est arbitraire. Elle ne peut pas rendre compte de ce qui est caractéristique du postmodernisme, puisque le postmodernisme est indifférent à la différenciation des médias traditionnels qui définissent les disciplines variées [qui le constituent] (littérature, peinture, photographie, architecture, etc.) (Van Alphen 1989 : 820).

Et encore ! Quand van Alphen argue de l'interdisciplinarité, il engage une discussion dont il ne tire pas les dernières conséquences : car ce sont les limites des disciplines elle-mêmes qui peuvent être remises en cause, et puis les savoirs, eux, sont ab initio transdisciplinaires. On devrait parler plutôt d'« in-disciplinaire » (Kremer 2011). Autant le vœu de l'« indisciplinarité » attirait les intellectuels de l'Est, avant tout comme projection épistémologique en couleurs de la culture médiatique américaine, autant un vœu similaire, prononcé en France, renvoyait au désenchantement post-idéologique et post-révolutionnaire, invitant à une attitude de recueillement intellectuel succédant au dévergondage intellectuel soixante-huitard, voire même au discours catastrophiste de la fonte du réel dans ses simulacres, chez Baudrillard et Cie. Un ordre du discours était en train de s'écrouler, mais en Europe de l'Est et en France, « l'ordre du discours » ne renvoyait pas aux mêmes discours. En France et en Europe de l'Est, on ne parlait pas des mêmes choses quand on disait « postmoderne $»$. 
MATEI

L'engouement pour le terme « postmodernisme » à l'Est s'explique peutêtre à partir du suffixe -isme qui renvoie aux avant-gardes, aux manifestes et notamment à l'époque de l'entre-deux-guerres où ils avaient pris naissance, et par conséquent à un certain type de publicité culturelle que les régimes communistes avaient toujours interdit, mais que le dernier dégel, à l'époque de Gorbatchev, permettait tant bien que mal. Dans la mesure où c'est le terme de « postmodernisme » plus que le postmoderne ou bien la postmodernité qui s'y impose, on est autorisé à parler d'un moment de modernité « itérable » (Derrida 1988 : 18) qui fait miroiter des lendemains qui chantent. Ainsi, la liberté à laquelle renvoie le « postmodernisme » est à la fois une ouverture, un à-venir, et le retour à la liberté d'avant les régimes communistes. En même temps, en France, le « postmoderne » désigne la progression d'un front maussade, l'envers des Trente glorieuses, une césure que l'on voulait surmontée aussi vite que possible : un cul-de-sac, dont peut-être la meilleure expression littéraire est de nos jours la trilogie de Virginie Despentes, Vernon Subutex (2015-2018).

Lyotard a raison de mettre à profit une méthodologie « indisciplinée », car en Europe de l'Ouest, le « postmoderne » noue trois moments : celui de la « dérégulation » médiatique, dans lequel le contrôle des médias passe, peu à peu, des mains gouvernements étatiques aux mains des stratèges marketing des compagnies multinationales ; le moment de la montée des droits de l'homme, alors que les mythes soviétiques de l'émancipation de l'homme et de la performance économique se révèlent définitivement compromis (surtout à la suite de Août 1968) ; enfin, moment de poussée consumériste, menée à l'Ouest comme à l'Est, quoique à deux vitesses. Or, toute approche partielle de ce moment risque de n'en faire que la source d'un repositionnement dans les mêmes cadres : on ne peut parler, par exemple, de « littérature postmoderne » sans parler des société(s) où elle se fait et se consomme, et des régimes de pouvoir où elle s'insère.

C'est au travers de l'in-disciplinarité qu'on arrive à saisir la portée méthodologique de la catégorie de « récit » au cours de l'enquête sur le postmoderne/ isme. Le défi que présuppose la mise en avant de ce concept, chez Lyotard, est de taille, car il réunit des domaines censés garder leur spécificité : littérature d'une part, puis philosophie, épistémologie, etc. Moins de vingt ans plus tard, il existe déjà des « cartographies » du postmodernisme littéraire, et l'une d'entre elles nous intéresse particulièrement. Il s'agit de l'ouvrage dirigé par Jan Bertens et Douwe Fokkema, International Postmodernism. Theory and Literary Practice, paru en 1997, qui retient chez Lyotard ce que deviendra une marque d'auteur, à savoir la « fin des métarécits » : 
Post-modern-east ou comment peut-on être « post-moderniste sans post-modernité »

En approchant le terme de « postmoderne » du point de vue historique, on découvre qu'il s'impose relativement tard en philosophie, à savoir en 1979, alors que Jean-François Lyotard publiait La Condition postmoderne (1979). Ce livre est devenu, même en dehors de la philosophie, l'ouvrage le plus connu concernant le postmoderne. Lyotard y caractérise le « postmoderne » comme « fin des métarécits $\gg$ (Welsch, Sandbothe $1997: 75$ - notre traduction)

Dans son chapitre consacré au «postmodernisme en France », Geert Lernout attire l'attention sur l'absence de ce terme des débats intellectuels français, mais tente aussi d'expliquer cette absence. Ce n'est pas à cause de l'ignorance que ce terme ne circule pas en France : son usage serait inutile, car le mot «moderne » en couvrirait la portée ${ }^{2}$. Les écrivains français en font usage seulement dans des textes destinés à un public étranger. Est-ce suffisant ? Car « moderne » ne saurait pas y suppléer en toute circonstance, ne serait-ce qu'à cause de nombreuses chronologies selon lesquelles on peut déplacer le début de l'époque moderne du XVIIe au XIXe siècles, de Charles Perrault à Charles Baudelaire.

En effet, avec son « postmoderne », Lyotard fait figure à part non seulement par rapport à son public, mais également auprès de ses collègues. Ni Foucault ni Deleuze, et Barthes non plus ne pensent jamais à discuter le « postmoderne ». L'affaire des « nouveaux philosophes $\gg$ y est sans doute pour quelque chose, puisque c'est précisément la French Theory (qu'ils représentent) que ceux-ci combattent durant la seconde partie des années 1970. Ce serait alors plutôt ces « nouveaux philosophes » qui pourraient se faire appeler « postmodernes », mais alors leur style, très peu ludique, voire pathétique chez Bernard HenriLévy, l'interdit. En définitive, pourquoi les maîtres penseurs décriés par les « nouveaux » adopteraient-ils un terme qui n'est pas de leur invention, alors que leur capital intellectuel est encore intact ? Les raisons de capital symbolique qui poussent le « postmodernisme » sur le devant de la scène nord-américaine font défaut en France.

\section{Post-Modern-East : un fantasme}

En Europe de l'Est, des régimes stagnants s'efforçaient de prolonger aussi longtemps que possible une idéologie qui avait révélé son incapacité à être mise en pratique. Par conséquent, les intellectuels de l'Est essayaient de vivre

$2 \ll$ The reason for this neglect of the term postmodernism is simple: for domestic use the term "modern" is quite sufficient to describe the same phenomena that are elsewhere called postmodern, which is illustrated by Jean-Paul Aron's decision to call his attack on the development of French thinking between 1945 and 1984, Les Modernes » (Leernout $1997: 353$ ) 
aux rythmes et dans les cadres des débats occidentaux, tout en censurant ce que, dans leurs propos, pouvait prêter à suspicion. Le moment postmoderne de l'Ouest, avec ses trois composantes, avait sans doute une force d'attraction immense. Dérégulation des médias, droits des hommes, intensification consommatrice, tous ces trois fils noués ensemble brossaient un tableau avenant, qui cachait pourtant des dessous : des inégalités, certes, mais surtout, et c'est que Lyotard s'emploie à souligner dans son livre, la dégradation du statut de l'intellectuel humaniste. A quoi bon des intellectuels dans une société de la concurrence sauvage, illimitée, tellement houellebecquienne ? Le paradoxe est aujourd'hui évident : les intellectuels humanistes de l'Est avaient été touchés par la magie d'un phénomène culturel dont la première conséquence allait être leur relégation.

Au fil des chapitres qui composent le tableau de la réception du « postmodernisme $\gg$ en Europe de l'Est, dans le livre de Hans Bertens, Douwe Fokkema, on est en droit d'en conclure qu'un débat sur le postmoderne commence ici durant les années 1980 et que, à quelques exceptions près, il concerne surtout la littérature tout en s'appuyant sur une construction critique américaine. Que le regard des Européens soit rivé aux Etats-Unis n’a rien d'étonnant. A défaut d'assister à des concerts de musique pop américaine et d'avoir un plein accès à Coca-Cola, à défaut de participer à des élections libres et de voyager à travers le monde, les intellectuels de l'Est pouvaient au moins parler de Beat poetry, pouvaient lire et traduire John Barth ou Kurt Vonnegut et penser avec Richard Rorty ou Ihab Hassan.

Là où la question postmoderne a pu mobiliser des ressources intellectuelles plus importantes, notamment en Yougoslavie et en Pologne, le terme désignait plutôt la fin d'une époque (moderne, socialiste) et possédait, ainsi, une charge dissidente. Par ailleurs, les prémices d'une pensée « post-moderne »y existent depuis des décennies. Le Tchèque Jindrich Chalupecký avait écrit, avant la mainmise de Staline sur la région, un livre sur la « fin de la modernité » dont le titre semble faire suite à l'idée de la « fin d'une civilisation » lancée plus tôt par Valéry, alors que Milan Suchomel écrit à son propos qu'il semble plutôt annoncer la thèse de la modernité inachevée de Habermas (Suchomel 1997 : 419).

Les contraintes qui ont pesé sur les champs culturels des pays de l'Est n'ont pas eu le même poids partout. Ainsi, il se peut que l'ouverture postmoderne a été plus large en Pologne puisque « la littérature n' $[y]$ avait guère connu le réalisme socialiste qui, dans d'autres pays slaves, en a empêché le développement » (Janaszek-Ivanicková 1997: 425). En Hongrie, la longue tradition de pensée de gauche enracine assure au débat un enracinement idéologique selon lequel le moment post-moderne est placé, en rétrospection, en 1968. Agnès Heller « considérant que la condition postmoderne est un sous-produit de 
l'aliénation et des mouvements politiques de 1968, (...) la lie à la dissolution des cultures de classe » et à la fin des idéologies (et de l'Histoire en tant que produit idéologique) (Szegedy-Maszák 1997 : 431). Enfin, la Slovénie est, parmi les pays de l'ex-Yougoslavie, le lieu privilégié où un débat à ce sujet peut s'enclencher à même dans le journal officiel Delo (Le Travail), en 1984, c'est toujours là que le philosophe Jakob Kos écrit sur la pensée post-moderne sous l'égide d'Auguste Comte, Marx et Nietzsche (Mozejko 1997 : 442). Il faut aussi remarquer une convergence des débats slaves : c'est à Novi Sad que, à la fin des années 1980, des intellectuels slovaques, dans la revue Novýzzivot, et serbes, dans Polja, publient des articles autour du postmodernisme. Dans une Yougoslavie qui n'avait jamais connu, elle non plus, la pression du réalisme socialiste, une revue en langue slovaque insère des textes théoriques sur le postmodernisme qui en font vraiment un moment de bilan théorique (Mozejko 1997 : 445). Parmi eux, le philosophe slovène Ales Erjavec signe l'essai Filozofia kritickej teorie a postmodernizmus (Erjavec 1989).

Au vu de ce très bref compte-rendu, on constate que, dans les années 1980, les débats sur le postmodernisme se donnent non seulement les enjeux permis par une censure variable, et de plus en plus lâche, et s'en tiennent pour l'essentiel à une pensée esthétique, sans dépasser, dans la plupart des cas, une sorte de fascination intellectuelle pour des discours que les intellectuels de l'Est ne pensent pas (pouvoir) acclimater dans leurs sociétés. Ce sont les pays dont les champs littéraires étaient moins endettés auprès de l'Union Soviétique qui ont le plus puisé dans les références américaines du terme (le cas de la Yougoslavie est le plus remarquable en ce sens). En dernière instance, le postmoderne devient une sorte de «préposition », selon Latour : «Il s'agit en effet d'une prise de position qui vient avant la proposition et qui décide de la façon dont on doit la saisir et qui constitue sa clef d'interprétation. » (Latour 2012 : 69). Le « postmoderne » fonctionne comme clé de lecture, parfois des mêmes textes que l'on avait lus autrement avant, et qui permet également de relire la dynamique culturelle nationale selon une indexation plus ou moins transatlantique.

Bien que les termes de « post-moderne » et « post-modernisme » aient engendré, dès le milieu des années 1980, un des plus importants débats intellectuels en Roumanie depuis le rejet du « réalisme socialiste $\gg$ au début des années 1960, la réception roumaine de La Condition postmoderne de JeanFrançois Lyotard, référence incontournable dans la bibliographie du « postmoderne », a été à la fois tardive et marginale, en termes d'influence et de traduction. Le livre a été traduit en 1993 et publié par une petite maison (réimprimé en 2003), alors que le contexte français de la parution du livre avait changé. 
MATEI

Paru à la fin des années 1970, le livre de J.-F. Lyotard surgit dans un contexte intellectuel différent de celui «théorique » déjà connu et partiellement discuté en Roumanie (structuralisme, nouvelle critique, « nouveau roman $\gg$ ). Il s'agit de l'émergence des « nouveaux philosophes », polémiques à l'encontre de toute théorie qui tente de légitimer un certain sens de l'Histoire, et notamment à l'encontre de l'idéologie qui justifiait encore la division politique de l'Europe et du monde (en gros, 9le « marxisme »). Or, ces jeunes intellectuels français étaient à la fois anti-totalitaires et dénonçaient les prétentions des théories de l'histoire à légitimer un monde et un homme nouveaux, quelles qu'en fussent les versions.

Un tel contexte, directement politique, ne pouvait pas être livré auprès du public roumain, de sorte que le peu d'intellectuels roumains qui ont participé à la campagne « post-moderniste » à partir du milieu des années 1980, et qui le connaissaient, ont dû passer sous silence les enjeux politiques du « post-modernisme $\gg$, sous peine de félonie idéologique flagrante. Le modèle institutionnel américain, en disposition « en parallèle », dans lequel universités et pouvoirs politiques évoluaient côte à côte sans heurts majeurs, les arrangeait bien plus que le modèle institutionnel français, « en série », où culture et politique participaient du même réseau de pouvoir. Ce sont alors plutôt les références apolitiques, visant l'histoire des formes et des procédés littéraires, qui permettent à ces intellectuels de publier nombre d'essais autour du postmodernisme. En philosophie, pour ces mêmes raisons, La Condition postmoderne bénéficie de peu de publicité. La pensée de Lyotard est une pensée sceptique de fin d'époque, tout en étant critique à la fois avec la temporalité ascendante de l'histoire hégélienne et marxienne, et avec la temporalité immédiate et cumulative du capitalisme.

Le « postmoderne » entre en Roumanie à travers les échos de la dispute Habermas - Lyotard, mais ce postmodernisme philosophique circule peu dans les milieux intellectuels. Creuser dans les détails de cette controverse aurait touché des points sensibles de l'actualité politique européenne, et la censure ne pouvait pas le permettre. C'est le postmodernisme littéraire qui a eu plus de chances à faire débat, et c'est ce qui s'est en effet passé : les littéraires roumains seront tous transis par un débat postmoderne qui durera une bonne dizaine d'années, avant et après l'écroulement du Mur de Berlin, avec plus d'entrain avant qu'après, car entre temps l'Histoire avait fait son boulot : après 1990, le postmodernisme n'avait plus, du moins selon sa lecture en termes littéraires, rien de subversif.

Par-delà la charnière politique de 1989, alors que le système communiste s'écroule, le débat « postmoderniste » continue à remuer les esprits tout en se tenant soigneusement à distance de présent historique trouble. En pleine 
Post-modern-east ou comment peut-on être « post-moderniste sans post-modernité »

époque de «transition », à peine libéré de la prison de l'austérité et des contraintes de toute sorte, l'intellectuel roumain avait du mal, encore une fois, à « vérifier » sur le terrain la « réalisation » de la postmodernité. Enfin, depuis la perspective plus restreinte de la dynamique transnationale de la littérature, la présence chétive de cet ouvrage de Lyotard (et de tout son œuvre au fond) dans le débat postmoderne s'explique aussi à partir de l'idée des « stratégies d'universalisation » discutées par Andrei Terian, par lesquelles les littératures périphériques revendiquent une place sur le devant de la scène mondiale. Il s'agit notamment de la stratégie «de compensation » : « une tentative d'enrayer une influence trop grande d'une littérature étrangère par la réorientation vers une autre littérature étrangère $\gg$ (Terian 2013), qui fait que les débats littéraires qu'ont menés les acteurs du champ littéraire roumain après le dégel des années 1960, pour la plupart « transférés » notamment depuis la culture littéraire française, demandaient à être remplacés par des produits d'importation différente. Il s'agissait, en l'occurrence, et pour la première fois en Roumanie, d'une importation culturelle américaine, importation de concepts et d'arguments aptes à se faire passer pour des « universaux » contemporains, et dont il était, en tant que tels, inutile de questionner l'enracinement culturel. De ce point de vue, on pourrait oser l'hypothèse que « la condition postmoderne » est, en Roumanie, un symptôme de la fin d'une méta-narration culturelle qui n'est pas tout simplement « moderne », mais plutôt française, et de l'ouverture vers l'horizon a priori illimité d'une multiplicité narrative capitaliste dont l'emprise est à la hauteur de sa capacité à simuler la liberté, d'origine nord-américaine.

En effet, dès la fin des années 1970, s'installe en France un éthos «mou », « apathique », un malaise dont les expressions théoriques avaient du mal à être transférées ailleurs, comme il s'était agi avec les théories réunies autour du structuralisme. Quand, en mai 1980, peu de temps après la mort de Barthes dont il avait été un ami proche, Antoine Compagnon écrit à peu près vingt pages sur l'œuvre d'Emil Cioran qui venait de publier Ecartèlement (le futur auteur des Antimodernes déclare avoir à peine découvert cette œuvre), il place l'actualité de Cioran et de son discours sceptique en tant que signe d'une « crise de l'énergie » que déclenche «la désuétude sans recours des narrations et des légitimations dont le siècle se soutenait naguère », renvoyant nommément à la Condition postmoderne (Compagnon $1980: 458$ ). Le terme « postmoderne » lui semble insignifiant, de sorte qu'il considère que le reprendre dans son texte serait inutile. On se donne plus de peine à nommer des réalités en train de naître que d'apposer des labels sur des réalités en train de s'essouffler. C'est comme $\mathrm{si}$ « postmoderne » n'était qu'une version anodine de dire la «crise de l'énergie », syntagme qui, pour Compagnon, garde toute l'ambiguité qu'il 
pouvait avoir pour un intellectuel français dont la vigilance ne manque pas à relier crise d'énergie fossile et crise d'énergie intellectuelle. La lecture du postmoderne en termes d'engagement affectif n'était pourtant pas l'apanage de Compagnon, qui devait avoir lu de Lyotard plus que la Condition postmoderne. En 1977, « Apathie de la théorie » est le titre du premier chapitre des Rudiments païens, un recueil d'articles publiés entre 1974 et 1976 . Lyotard y propose dans son essai une «psychologie » de la théorie : « normalement, la théorie s'accompagne d'un affect spécifique, la conviction $\gg$ (Lyotard 1977 : 77) : moins de conviction, donc moins de théorie. Quand ils écrivent sur la réédition de La Société de consommation de Jean Baudrillard (qui reparaît chez Gallimard en 1974), Pascal Bruckner et Alain Finkielkraut en arrivent à ce même vocabulaire. Il est vrai que Bruckner et Finkielkraut battent en brèche la critique de la société de consommation entreprise par Baudrillard, puisqu'ils y prennent l'indifférence par son tenant destructeur de pathos, et la transforme en moteur d'émancipation, car c'est l'indifférence qui annule « l'exigence de l'identité » qui sévit dans les sociétés modernes (Bruckner, Finkielkraut, 1975 : 473). L'homme postmoderne pourrait alors se féliciter d'être devenu « indifférent $\gg$ aux valeurs qui entravaient son développement. Cette approche, qui érige l'indifférence (et peut-être aussi la « neutralité » barthésienne ?) au rang de méta-affect, présente toutefois un vice théorique majeur : si l'aliénation généralisée peut mener à l'indifférence généralisée en tant que liberté de se passionner de tout (elle se recharge ainsi en pathos), mais individuellement, par la « cause efficiente » de la consommation, ce pathos de l'indifférence échappe à la nomination et ne crée plus de concept. Il renaît, certes, mais en dehors du concept, et par conséquent aussi au-delà du politique. Il devient une addiction, comme le jeu, qui s'accroche au mouvement même de capitalisation auquel tous les autres buts sont subordonnés. La défection de la « conviction » n'a de cesse à être déplorée : un dernier ouvrage sur ce sujet vient de paraître en France, Nous ne savons plus croire, ce qui montre que l'impasse de la désaffection postmoderne n'a pas vraiment été dépassée (Riquier 2020).

\section{Les deux bornes du débat postmoderne en Roumanie : 1986, 1995}

On serait tentés de croire que la libération de la parole aurait contribué, après décembre 1989, à enrichir le débat « postmoderne » en Roumanie, mais il n'en fut rien. Par rapport au numéro consacré à ce sujet au début de 1986 - il s'agissait d'une revue de théorie littéraire intitulée Caiete critice, de Bucarest -, celui de 1995, de la revue Euresis (à l'origine une revue qui publiait des articles en langues étrangères, Cahiers roumains d'études littéraires), qui clôt symboliquement le «moment postmoderne », le lecteur trouve peu de différences. 
Grosso modo, une fois la censure tombée, les intellectuels ont vite découvert que, en matière de littérature, ils n'avaient pas grand-chose à changer à ce qu' ils en disaient alors qu'elle était active.

Le premier changement tient aux langues d'usages : en 1995, la langue des articles n'est plus le roumain, mais l'anglais et le français, accessoirement l'allemand. La langue dominante reste pourtant le français, choix qui s'explique par la francophonie de la plupart des auteurs. Le passage du roumain vers des langues occidentales signale encore que les intellectuels roumains avaient le complexe de la précarité des échanges avec leurs collègues de l'Ouest, et qu'ils étaient désireux de parler non seulement leur langage (le langage « postmoderne »), mais aussi leur langue. Le sentiment d'appartenir à la périphérie européenne n'aura fait, après 1989 , que s'intensifier.

Le dossier de 1995 est plus fourni que celui de 1986, comme preuve peutêtre qu'on vit un moment de «plénitude », alors que, dix ans auparavant, la pénurie imposée par le régime de Ceausescu visait le papier aussi. Mais il y a peu de noms étrangers en 1995, et même Lyotard, dont la Condition postmoderne venait d'être traduite, en est absent. C'est comme si, une fois la liberté conquise, toute raison de subversion - puisque La Condition postmoderne était un texte subversif par rapport au nombre d'institutions modernes - se fût évanouie, comme s'il n'y avait plus de pouvoir qu'on devrait interroger. Larrivée des « multiplicités » que Lyotard énumérait dans son rapport de 1979 était arrivée, mais personne ne semblait les examiner : «multiplication des machines informationnelles », « entreprises multinationales », des « tâches de recherche », enfin, « des langages » (Lyotard 1979b : 12, 16, 67, 71). L'on ne savait pas, et on ne voulait pas savoir si ces « multiplicités » étaient bonnes ou mauvaises. Une fois les totalitarismes politiques vaincus, on s'y laissait aller sans les interroger : les « multiplicités » s'opposaient à la stagnation, le Multiple à l'Un, le mouvement à la stase : c'était l'《extase » postmoderne.

Si la plupart des articles qui composaient le numéro sur le postmodernisme de 1995 auraient pu paraître avant 1990, il y en avait au moins une exception : c'est l'avant-propos de Mircea Martin, « D'un postmodernisme sans rivages et d'un postmodernisme sans postmodernité $\gg$ (Martin 1995).

Tout comme Bertens et Fokkema deux ans plus tard, Martin commence par se poser la question de l'actualité du postmodernisme : « Le postmodernisme est-il un problème dépassé de la culture contemporaine ? » La question était en quelque sorte rhétorique, mais elle posait aussi le problème d'une époque où la vitesse de passage des historicités se serait tellement accélérée qu’on se demandait si elle n'arriverait pas à anéantir l'objet dont elle n'est qu'une propriété dynamique. Il y a toutefois un côté sérieux de la question : si le problème « est dépassé », c'est peut-être que le moment de la « révolution » de 1989 l'aurait 
en quelque sorte « résolu » d'emblée, par la chute du régime communiste. Ce régime avait été, Martin remarque, celui d'une modernité contraignante : réalisme socialiste et dénationalisation dans les années 1950, puis « épuisement du mythe communiste $\gg$ (Martin $1995: 8$ ).

Martin se pose donc en marge du « postmodernisme », et c'est depuis ce point de vue qu'il constate comment une littérature « postmoderniste » émergeait en Roumanie communiste, sans lien aucun avec l'« infrastructure », « comme pour contrarier la thèse marxiste, tant de fois vérifiée par les historiens, de la détermination de la superstructure - de la culture, donc - par une base économique. $\gg(8)$. De concert avec Foucault, avec Compagnon ou avec Bruckner-Fienkielkraut, Martin entrevoit un ethos « postmoderne », mais le « désenchantement » roumain avait une autre source que celui français : il s'agissait des échecs cuisants de la société communiste, et non capitaliste, qui entraînaient une « exaspération provoquée par le modernisme » (8), et non pas la « postmodernité ». Les écrivains roumains se serait rabattus sur ce terme américain par dépit, par ennui, comme le faisait Madame Bovary avec ses amants : pour fuir la monotonie.

La seconde raison de la pénétration d'un ethos postmoderne en Roumanie est, enchaîne Martin, d'ordre culturel : la nouvelle génération d'écrivains - les « quatre-vingt-huitards »- n'est plus francophone, « comme celle qui l'avait précédée », mais anglophone, regardant « du côté de l’Amérique » (9). Martin ne voit pas dans cette description aucun « présupposé $\gg$, si ce n'est la « fascination pour la littérature underground et la contre-culture $\gg(9)$. Certes, il a raison, mais beaucoup plus loin qu'il ne pense : il s'agissait de la mondialisation culturelle allant de pair avec la globalisation économique, dont le cours n'avait été que ralenti, mais non entravé par les régimes totalitaires. Sur ce point, le récit d'un passé traumatique pousse Martin à oublier jusqu'au premier dossier consacré au postmodernisme en plein régime Ceausescu.

Globalisation et mondialisation allaient leur train, malgré la récession économique : s'il y a eu un sentiment d'isolement au niveau d'une couche intellectuelle qui devait subir les rigueurs parfois extrêmes de la pénurie, les années 1950 n'étaient pourtant qu'un souvenir. Il y eut, à partir de la fin des années 1960, à travers les médias et leurs usages permis ou (apparemment) clandestins, la constitution d'une infrastructure « imaginaire » que la révolution roumaine télévisée, sujet de réflexion de Jean Baudrillard entre autres, avait d'ailleurs résumée (Petrovszky, Țichindeleanu 2009). Si les postmodernes roumains avaient été subversifs par rapport à « l'autarcie et au traditionalisme rhétorique » (9), c'est comme si la chute du régime et l'adhésion immédiate de la plupart des intellectuels roumain aux «valeurs européennes » les avaient dispensés de l'exercice critique et créatif de la subversion. Et c'est comme si 
« le traditionalisme rhétorique » n’avait aucune autre source que le discours politique officiel, celui immédiatement officiel. Comme si les pouvoirs qui avaient poussé à l'ombre du Pouvoir et s'y étaient épanouis d'autant mieux que ce Pouvoir prétendait tout contenir et étouffer n'y étaient pour rien dans cette rhétorique que les postmodernes venaient interroger.

Le moment postmoderne est un moment de convergence ; un nom qui achève une époque, aux Etats-Unis, immédiatement postérieure à la Seconde Guerre mondiale, un nom qui ouvre une autre époque, en Europe de l'Est (et puis ailleurs : par exemple, mais d'une manière particulière, en Chine), et c'est dans cet écart chronologique que résident les inégalités que nous vivons aujourd'hui, et les libertés dont certains d'entre nous profitent. Que le même Mircea Martin se plaigne du discrédit jeté sur les études littéraires, aujourd'hui, n’a rien d'étonnant : un même processus qui muait, dans les années 1980, les idéologies qui avaient animé nombre d'intellectuels et de groupes sociaux avant 1968 en simples « récits » à effets illo- et per-locutionnaires, rabaissait après 1989, à l'Est, les discours de vérité qu'avaient été jadis l'histoire de la littérature nationale, la critique « de la conscience » ou bien le « critère esthétique » pour évaluer les textes littéraires, au rang des mythes dont les héros avaient ignoré la seule réalité « performative ». Le temps est venu, enfin, pour penser le post-modern-east au-delà des fantasmes qui l'ont enclenché, l'ont gonflé et l'ont abandonné ensuite à sa surface de papier glacé qui s'effrite au plus petit toucher.

\author{
Alexandru Matei \\ alexandru.matei@unitbv.ro \\ “Transilvania” University \\ ROMANIA
}

\title{
Bibliographie
}

Bruckner, P., Finkielkraut, A. 1975. Passions de l'indifférence. - Critique, Mai, no 336, 465-488.

Bruckner, P., Finkielkraut, A. 1980. Déscolariser l'intelligentsia. - Le Débat, 4 (septembre), 16-18. https://doi.org/10.3917/deba.004.0016

Calganio, A. 1998. Action, Passo et Creatio in the Endliche und Ewige Philosophie of Edith Stein. A Poetico-Personal Response to the Challenges of Postmodernity. - A.-T. Tymieniecka, ed., Acta Husserliana LII, https://link.springer.com/ chapter/10.1007/978-94-017-2604-7_20 (25.04.2021).

Derrida, J. 1988. Limited Inc. Evanston: Northwestern University Press.

Erjavec, A. 1989. Filozofia kritickej teorie a postmodernizmus. - Novýživot, 41, 633$637 ; 42,738-744$. 
MATEI

Felsi, R. 2015. The Limits of Critique. Chicago: University of Chicago Press.

Howe, I. 1959. Mass Society and Post-Modern Fiction. - Partisan Review, XXVI, http:// www.amerlit.com/documents/MASS_SOCIETY_AND_POSTMODERN_ FICTION.pdf (12.03.2020).

Janaszek-Ivanicková, H. 1997. Postmodernism in Poland. - H. Bertens, D. Fokkema, International Postmodernism. Theory and Literary Practice 1997. Amsterdam, Philadelphia: John Benjamins Publishing Company, 423-428.

Kremer, N. 2011. La littérature en mal de discipline? - Fabula LHT, mai, https://www. fabula.org/lht/8/ (25.04.2021).

Latour, B. 2012. Enquête sur les modes d'existence. Une anthropologie des Modernes. Paris : La Découverte.

Lyotard, J.-F. 1977. Rudiments païens. Genre dissertatif. Paris : UGE.

Lyotard, J.-F. 1979a. Rapport sur les problèmes du savoir dans les sociétés industrielles les plus développées, fait au Président du Conseil des Universités auprès du Gouvernement du Québec. Québec, deuxième tirage. Bibliothèque Nationale du Québec.

Lyotard, J.-F. 1979b. La Condition postmoderne. Paris: Minuit.

Lyotard, J.-F. 1984a. Le postmoderne expliqué aux enfants. Paris: Galilée.

Lyotard, J.-F. 1984b. Entretien avec Georges Van Den Abbeele. - Diacritics, 14 (3), 15-21. https://doi.org/10.2307/464841

Lyotard, J.-F. 1986. Réponse à la question: Qu'est-ce que le postmoderne? [Răspuns la întrebarea: ce este postmodernul?]. - Caiete critice, 1-2, 186.

Martin, M. 1995. D’un postmodernisme sans rivages et d'un postmodernisme sans postmodernité. - Euresis. Revue roumaine d'études littéraires, 1, 3-13.

Mozejko, E. 1997. Postmodernism in the Literatures of Former Yugoslavia. - H. Bertens, D. Fokkema, International Postmodernism. Theory and Literary Practice 1997. Amsterdam, Philadelphia: John Benjamins Publishing Company, 441-446.

Petrovszky, K., Tichindeleanu, O. 2009. Revoluția Română televizată. Contribuții la istoria culturală a mediilor, Cluj-Napoca: Ideea Design \& Print.

Riquier, C. 2020. Nous ne savons plus croire. Paris : Desclée de Brouwer.

Ruffel, L. 2016. Brouhaha. Les mondes du contemporain. Paris : Verdier.

Suchomel, M. 1997. Postmodernism in Czech Literature. - H. Bertens, D. Fokkema, International Postmodernism. Theory and Literary Practice 1997. Amsterdam, Philadelphia: John Benjamins Publishing Company, 419-422.

Szegedy-Maszák, M. 1997. Postmodern Literature in Hungary. - H. Bertens, D. Fokkema, International Postmodernism. Theory and Literary Practice 1997. Amsterdam, Philadelphia: John Benjamins Publishing Company, 429-434.

Terian, A. 2013. National Literature, World Literatures, and Universality in Romanian Cultural Criticism 1867-1947. - CLCWeb: Comparative Literature and Culture, 15.5. https://doi.org/10.7771/1481-4374.2344

Van Alphen, E. 1989. Review: The Heterotopian Space of the Discussions on Postmodernism. (A Poetics of Postmodernism: History, Theory, Fiction by Linda Hutcheon; After the Great Divide: Modernism, Mass Culture and Postmodernism by 
Post-modern-east ou comment peut-on être « post-moderniste sans post-modernité »

Andreas Huyssen; Postmodernist Fiction by Brian McHale.) - Poetics Today, 10 (4), 819-839. https://doi.org/10.2307/1772812

Welsch, W., Sandbothe, M. 1997. Postmodernity as a Philosophical Concept. - H. Bertens, D. Fokkema, International Postmodernism. Theory and Literary Practice 1997. Amsterdam, Philadelphia: John Benjamins Publishing Company, 75-88. 